\title{
Traveling wave solutions of the time-delayed generalized Burgers-type equations
}

\author{
Bo Tang ${ }^{1,2^{*}}$, Yingzhe Fan ${ }^{1,3}$, Xuemin Wang $^{4}$, Jixiu Wang ${ }^{1}$ and Shijun Chen ${ }^{1}$
}

${ }^{*}$ Correspondence:
tangbo0809@163.com
${ }^{1}$ School of Mathematics
and Computer Science,
Hubei University
of Arts and Science,
Xiangyang 441053, China
Full list of author information
is available at the end of the
article
article

\begin{abstract}
Background: Recently, nonlinear time-delayed evolution equations have received considerable interest due to their numerous applications in the areas of physics, biology, chemistry and so on.

Methods: In this paper, we obtain traveling wave solutions by using the extended $\left(\frac{G^{\prime}}{G}\right)$-expansion method.

Results: Based on the method, we get many solutions of the time-delayed generalized Burgers-type equations.

Conclusions: The results reveal that the extended $\left(\frac{G^{\prime}}{G}\right)$-expansion method is direct, effective and can be used for many other nonlinear time-delayed evolution equations.

Keywords: Nonlinear time-delayed evolution equations, Extended $\left(\frac{G^{\prime}}{G}\right)$-expansion method, Traveling wave solution
\end{abstract}

\section{Background}

In recent years, theory and numerical analysis of nonlinear time-delayed evolution equations have received considerable interest due to their numerous applications in the areas of physics, biology, chemistry and so on. For better studying the nonlinear physical phenomena of nonlinear time-delayed evolution equations, the solution is much involved. In the past, several analytical and numerical methods have been used to find solutions of nonlinear partial differential equations, such as homotopy perturbation method (Kumar and Singh 2009; Kumar et al. 2012; He 1999), Laplace transform (Kumar 2014), variational iteration method (He 1997; He and Wu 2007; Tang et al. 2014), residual power series method (RPSM for short) (Kumar et al. 2016b; Yao et al. 2015), auxiliary equation method (Sirendaoreji 2003; Tang et al. 2016; Yomba 2004), homotopy analysis method (Yin et al. 2015; Kumar et al. 2016a), $\left(\frac{G^{\prime}}{G}\right)$-expansion method (Wang et al. 2008; Zhang et al. 2010; Tang et al. 2011; Islam et al. 2014; Khan and Akbar 2014) and so on.

In this paper, we apply the extended $\left(\frac{G^{\prime}}{G}\right)$-expansion method to obtain traveling wave solutions of the following time-delayed generalized Burgers-type equations (Kar et al. 2003): 
- The time-delayed generalized Burgers equation:

$$
\tau v_{t t}+v_{t}+p v^{s} v_{x}-v_{x x}=0 .
$$

where $p, s$ are constants and $\tau$ is a time-delayed constant.

- The time-delayed generalized Burgers-Fisher equation:

$$
\tau v_{t t}+\left(1-\tau f_{v}\right) v_{t}=v_{x x}-p v^{s} v_{x}+f(v), \quad f(v)=q v\left(1-v^{s}\right) .
$$

This paper is organized as follows: in "Methods" section, the main steps of extended $\left(\frac{G^{\prime}}{G}\right)$-expansion method for obtaining traveling wave solutions of nonlinear time-delayed evolution equation are given. In "Results" section, we construct traveling solutions of the time-delayed generalized Burgers-type equation. Some conclusions are given in "Conclusions" section.

\section{Methods}

Considering the following nonlinear evolution equation:

$$
P\left(v, v_{t}, v_{x_{1}}, v_{x_{2}}, v_{x_{3}}, \ldots\right)=0,
$$

where $P$ is a polynomial in $v=v\left(x_{1}, x_{2}, x_{3}, \ldots, t\right)$ and its various partial derivatives.

Step 1 By means of the traveling wave transformation

$$
v=V(\eta), \quad \eta=k_{1} x_{1}+k_{2} x_{2}+k_{3} x_{3}+\cdots+h t+\eta_{0},
$$

where the coefficients $k_{i}, h$ are constants. Equation (1) can be transformated as follows:

$$
P\left(V(\eta), V^{\prime}(\eta), V^{\prime \prime}(\eta), \ldots\right)=0 .
$$

Step 2 We suppose that the Eq. (3) has the following solution:

$$
V(\eta)=\sum_{l=-n}^{n} a_{l}\left(\frac{G^{\prime}}{G}\right)^{l},
$$

where $a_{l}$ are constants to be determined later, and $G(\eta)$ satisfies the following equation:

$$
G^{\prime \prime}(\eta)+\alpha G^{\prime}(\eta)+\beta G(\eta)=0,
$$

where $\alpha$ and $\beta$ are arbitrary constants. Based on Eq. (5), we have

$$
\frac{G^{\prime}(\eta)}{G(\eta)}= \begin{cases}-\frac{\alpha}{2}+\frac{\sqrt{\alpha^{2}-4 \beta}}{2}\left(\frac{C_{1} \sinh \left(\frac{\sqrt{\alpha^{2}-4 \beta} \eta}{2}\right)+C_{2} \cosh \left(\frac{\sqrt{\alpha^{2}-4 \beta} \eta}{2}\right)}{C_{1} \cosh \left(\frac{\sqrt{\alpha^{2}-4 \beta} \eta}{2}\right)+C_{2} \sinh \left(\frac{\sqrt{\alpha^{2}-4 \beta} \eta}{2}\right)}\right), & \alpha^{2}-4 \beta>0, \\ -\frac{\alpha}{2}+\frac{\sqrt{4 \beta-\alpha^{2}}}{2}\left(\frac{-C_{1} \sin \left(\frac{\sqrt{4 \beta-\alpha^{2} \eta}}{2}\right)+C_{2} \cos \left(\frac{\sqrt{4 \beta-\alpha^{2}} \eta}{2}\right)}{C_{1} \cos \left(\frac{\sqrt{4 \beta-\alpha^{2}} \eta}{2}\right)+C_{2} \sin \left(\frac{\sqrt{4 \beta-\alpha^{2}} \eta}{2}\right)}\right), & \alpha^{2}-4 \beta<0 . \\ \frac{C_{2}}{C_{1}+C_{2} \eta}-\frac{\alpha}{2}, & \alpha^{2}-4 \beta=0 .\end{cases}
$$


Step 3 Determine the degree $n$ in Eq. (3) by use of homogenous balanced principle (Abdel Rady et al. 2010; Fan and Zhang 1998a, b; Senthilvelan 2001; Zhao and Tang 2002; Fan 2000; Eslami et al. 2014), namely balancing the highest order derivatives and nonlinear terms in Eq. (3).

Step 4 Substituting Eqs. (4) and (5) into Eq. (3) and clearing the denominator and collecting all terms with the same order of $\left(\frac{G^{\prime}}{G}\right)$ together, then setting each coefficient of $\left(\frac{G^{\prime}}{G}\right)^{l}$ to zero, we get a system of under-determined algebraic equations for $k_{i}, h$ and $a_{l}$.

Step 5 Solving the algebraic equations in Step 4 by Maple (www.maplesoft.com), we can finally get traveling wave solutions of Eq. (1).

\section{Results}

In this section, we apply the extended $\left(\frac{G^{\prime}}{G}\right)$-expansion method to obtain traveling wave solutions of the time-delayed generalized Burgers-type equations.

\section{Solutions to the time-delayed generalized Burgers equation}

We consider the following time-delayed generalized Burgers equation:

$$
\tau v_{t t}+v_{t}+p v^{s} v_{x}-v_{x x}=0 .
$$

By using transformations $v(x, t)=V(\eta)$ and $\eta=k(x-\omega t)$, Eq. (6) can be reduced as follows:

$$
\left(\tau \omega^{2}-1\right) k^{2} V^{\prime \prime}-k \omega V^{\prime}+p k V^{s} V^{\prime}=0 .
$$

Balancing $V^{\prime \prime}$ with $V^{s} V^{\prime}$ gives $n=\frac{1}{s}$ which is not an integer as $s \neq 1$. So we use a transformation $V=W^{\frac{1}{s}}$ to change Eq. (7) into the form:

$$
\left(\tau \omega^{2}-1\right) k^{2}\left[W^{\prime \prime} W+\left(\frac{1}{s}-1\right) W^{\prime 2}\right]-k \omega W^{\prime} W+p k W^{\prime} W^{2}=0 .
$$

We suppose that the solutions of (8) have the form (4) and (5), so

$$
\begin{aligned}
W^{\prime}(\eta)= & \sum_{l=-n}^{n} a_{l}\left(\frac{G^{\prime}}{G}\right)^{l-1} \frac{G^{\prime \prime} G-G^{\prime 2}}{G^{2}} \\
= & -\sum_{l=-n}^{n} a_{l}\left(\frac{G^{\prime}}{G}\right)^{l-1}\left[\beta+\alpha\left(\frac{G^{\prime}}{G}\right)+\left(\frac{G^{\prime}}{G}\right)^{2}\right], \\
W^{\prime \prime}(\eta)= & \sum_{l=-n}^{n} a_{l}\left(\frac{G^{\prime}}{G}\right)^{l-2}\left[\beta+\alpha\left(\frac{G^{\prime}}{G}\right)+\left(\frac{G^{\prime}}{G}\right)^{2}\right]^{2} \\
& +\sum_{l=-n}^{n} a_{l}\left(\frac{G^{\prime}}{G}\right)^{l-1}\left[\alpha+2\left(\frac{G^{\prime}}{G}\right)\right]\left[\beta+\alpha\left(\frac{G^{\prime}}{G}\right)+\left(\frac{G^{\prime}}{G}\right)^{2}\right] .
\end{aligned}
$$

From above two equations, we can get the degrees of $W^{\prime \prime} W$ and $W^{\prime} W^{2}$ are $2 n+2$ and $3 n+1$ respectively. Balancing $W^{\prime \prime} W$ and $W^{\prime} W^{2}$ in Eq. (8) yields $2 n+2=3 n+1$, namely $n=1$. Therefore Eq. (8) have the following solutions: 


$$
W(\eta)=\sum_{l=-1}^{1} a_{l}\left(\frac{G^{\prime}}{G}\right)^{l} .
$$

Substituting Eqs. (9) and (5) into Eq. (8), we get a set of under-determined algebraic equations for $a_{l}(l=0, \pm 1), k, \omega, \alpha$ and $\beta$.

$$
\begin{aligned}
& \left(\frac{G^{\prime}}{G}\right)^{4}: 2\left(\tau \omega^{2}-1\right) k a_{1}^{2}+\frac{k\left(\tau \omega^{2}-1\right)(1-s) a_{1}^{2}}{s}-p a_{1}^{3}=0, \\
& \left(\frac{G^{\prime}}{G}\right)^{3}: 3\left(\tau \omega^{2}-1\right) k \alpha a_{1}^{2}+2\left(\tau \omega^{2}-1\right) k a_{1} a_{0} \\
& +\frac{2 k \alpha\left(\tau \omega^{2}-1\right)(1-s) a_{1}^{2}}{s}+\omega a_{1}^{2}-2 p a_{0} a_{1}^{2}-p \alpha a_{1}^{3}=0, \\
& \left(\frac{G^{\prime}}{G}\right)^{2}: \frac{\left(\tau \omega^{2}-1\right) k(1-s)\left(-2 a_{1} a_{-1}+2 a_{1}^{2} \beta+a_{1}^{2} \alpha^{2}\right)}{s}+p a_{0}^{2} a_{1}(1+2 \alpha) \\
& +\left(\tau \omega^{2}-1\right) k\left(\alpha^{2}+2 \beta\right) a_{1}^{2}+3\left(\tau \omega^{2}-1\right) k \alpha a_{1} a_{0}-p a_{1}^{3} \beta \\
& +2\left(\tau \omega^{2}-1\right) k a_{1} a_{-1}+\omega a_{0} a_{1}+\omega a_{1}^{2} \alpha-p a_{1}^{2} a_{-1}=0, \\
& \left(\frac{G^{\prime}}{G}\right)^{1}: \frac{\left(\tau \omega^{2}-1\right) k(1-s)\left(-4 a_{-1}+2 a_{1} \beta\right) a_{1} \alpha}{s}-p a_{1} \alpha\left(a_{1} a_{-1}+a_{0}^{2}\right) \\
& +\left(\tau \omega^{2}-1\right) k a_{1}\left(a_{1} \alpha \beta+4 a_{-1} \alpha+a_{0} \alpha^{2}+2 a_{0} \beta\right) \\
& -2 p a_{0} a_{1}^{2} \beta+\omega a_{1}\left(a_{0} \alpha+a_{1} \beta\right)=0, \\
& \left(\frac{G^{\prime}}{G}\right)^{0}:\left(\tau \omega^{2}-1\right) k\left(4 a_{-1} a_{1} \beta+2 a_{-1} a_{1} \alpha^{2}+a_{0} a_{1} \beta \alpha+a_{-1} a_{0} \alpha\right) \\
& +\left(a_{-1}-a_{1} \beta\right)\left(a_{-1} a_{1}+a_{0}^{2}\right) p-\omega a_{0} a_{-1}-\omega a_{0} a_{1} \beta \\
& +\frac{\left(\tau \omega^{2}-1\right) k(1-s)}{s}\left(-4 a_{-1} a_{1} \beta-2 a_{-1} a_{1} \alpha^{2}+a_{-1}^{2}+a_{1}^{2} \beta^{2}\right)=0, \\
& \left(\frac{G^{\prime}}{G}\right)^{-1}:\left(\tau \omega^{2}-1\right) k\left(4 a_{-1} a_{1} \alpha \beta+2 a_{-1} a_{0} \beta+a_{-1} a_{0} \alpha^{2}+a_{-1}^{2} \alpha\right) \\
& -\omega a_{-1}\left(a_{-1}+a_{0} \alpha\right)+p a_{-1}^{2} a_{1} \alpha+p a_{-1} a_{0}\left(2 a_{-1}+a_{0} \alpha\right) \\
& +\frac{\left(\tau \omega^{2}-1\right) k \alpha(1-s)}{s}\left(-4 a_{-1} a_{1} \beta+2 a_{-1}^{2}\right)=0, \\
& \left(\frac{G^{\prime}}{G}\right)^{-2}: \frac{\left(\tau \omega^{2}-1\right) k a_{-1}(1-s)}{s}\left(2 a_{-1} \beta-2 a_{1} \beta^{2}+a_{-1} \alpha^{2}\right) \\
& -\omega a_{-1}\left(a_{-1} \alpha+a_{0} \beta\right)+p a_{-1}^{2}\left(2 a_{0} \alpha+a_{1} \beta\right)+p a_{-1}^{3}+p a_{0}^{2} a_{-1} \beta \\
& +\left(\tau \omega^{2}-1\right) k\left(2 a_{-1} a_{1} \beta^{2}+3 a_{-1} a_{0} \alpha \beta+2 a_{-1}^{2} \mu+a_{-1}^{2} \alpha^{2}\right)=0, \\
& \left(\frac{G^{\prime}}{G}\right)^{-3}: \frac{2\left(\tau \omega^{2}-1\right) k a_{-1}^{2} \alpha \beta(1-s)}{s}+\left(\tau \omega^{2}-1\right) k a_{-1} \beta\left(2 a_{0} \beta+3 a_{-1} \alpha\right) \\
& -\omega a_{-1}^{2} \beta+p a_{-1}^{2}\left(a_{-1} \alpha+2 a_{0} \beta\right)=0, \\
& \left(\frac{G^{\prime}}{G}\right)^{-4}: \frac{\left(\tau \omega^{2}-1\right) k a_{-1}^{2} \beta^{2}(1-s)}{s}+2\left(\tau \omega^{2}-1\right) k a_{-1}^{2} \beta^{2}+p a_{-1}^{3} \beta=0 .
\end{aligned}
$$

Solving this algebraic equations by Maple, we can obtain the two results: 


\section{Case 1}

$$
\begin{aligned}
& a_{-1}= \pm \frac{(s+1) \beta \omega}{p \sqrt{\alpha^{2}-4 \beta}}, \quad a_{0}= \pm \frac{\alpha(s+1) \omega}{2 p \sqrt{\alpha^{2}-4 \beta}}+\frac{(s+1) \omega}{2 p}, \\
& a_{1}=0, \quad k=\mp \frac{s \omega}{\left(\tau \omega^{2}-1\right) \sqrt{\alpha^{2}-4 \beta}}
\end{aligned}
$$

where $\alpha, \beta$ and $\omega$ are arbitrary constants.

\section{Case 2}

$$
\begin{aligned}
& a_{-1}=0, \quad a_{0}= \pm \frac{\alpha(s+1) \omega}{2 p \sqrt{\alpha^{2}-4 \beta}}+\frac{(s+1) \omega}{2 p} \\
& a_{1}= \pm \frac{(s+1) \omega}{p \sqrt{\alpha^{2}-4 \beta}}, \quad k= \pm \frac{s \omega}{\left(\tau \omega^{2}-1\right) \sqrt{\alpha^{2}-4 \beta}}
\end{aligned}
$$

where $\alpha, \beta$ and $\omega$ are arbitrary constants.

Using Eqs. (9) and (10), we obtain the following solution of Eq. (6):

$$
\nu_{1}(\eta)=\left[ \pm \frac{\alpha(s+1) \omega}{2 p \sqrt{\alpha^{2}-4 \beta}}+\frac{(s+1) \omega}{2 p} \pm \frac{(s+1) \beta \omega}{p \sqrt{\alpha^{2}-4 \beta}}\left(\frac{G^{\prime}}{G}\right)^{-1}\right]^{\frac{1}{s}}
$$

where $\eta=\mp \frac{s \omega}{\left(\tau \omega^{2}-1\right) \sqrt{\alpha^{2}-4 \beta}}(x-\omega t)$.

Based on Eqs. (9) and (11), we get the solution of Eq. (6) as follows:

$$
\nu_{2}(\eta)=\left[ \pm \frac{\alpha(s+1) \omega}{2 p \sqrt{\alpha^{2}-4 \beta}}+\frac{(s+1) \omega}{2 p} \pm \frac{(s+1) \omega}{p \sqrt{\alpha^{2}-4 \beta}}\left(\frac{G^{\prime}}{G}\right)\right]^{\frac{1}{s}}
$$

where $\eta= \pm \frac{s \omega}{\left(\tau \omega^{2}-1\right) \sqrt{\alpha^{2}-4 \beta}}(x-\omega t)$.

Substituting the general solutions of Eq. (5) into Eq. (12), we have two kinds of travelling wave solutions as follows:

When $\alpha^{2}-4 \beta>0$,

$v(x, t)=\left[\frac{(s+1) \omega}{p \sqrt{\alpha^{2}-4 \beta}}\left(\frac{\sqrt{\alpha^{2}-4 \beta} \pm \alpha}{2} \pm \frac{2 \beta}{-\alpha+\sqrt{\alpha^{2}-4 \beta}\left(\frac{C_{1} \sinh \frac{\sqrt{\alpha^{2}-4 \beta}}{2}+C_{2} \cosh \frac{\sqrt{\alpha^{2}-4 \beta} \eta}{2}}{C_{1} \cosh \frac{\sqrt{\alpha^{2}-4 \beta}}{2}+C_{2} \sinh \frac{\sqrt{\alpha^{2}-4 \beta} \eta}{2}}\right)}\right)\right]^{\frac{1}{s}}$,

where $\eta=\mp \frac{s \omega}{\left(\tau \omega^{2}-1\right) \sqrt{\alpha^{2}-4 \beta}}(x-\omega t)$.

When $\alpha^{2}-4 \beta<0$,

$v(x, t)=\left[\frac{(s+1) \omega}{p \sqrt{\alpha^{2}-4 \beta}}\left(\frac{\sqrt{\alpha^{2}-4 \beta} \pm \alpha}{2} \pm \frac{2 \beta}{-\alpha+\sqrt{4 \beta-\alpha^{2}}\left(\frac{-C_{1} \sin \frac{\sqrt{4 \beta-\alpha^{2}} \eta}{2}+C_{2} \cos \frac{\sqrt{4 \beta-\alpha^{2} \eta}}{2}}{C_{1} \cos \frac{\sqrt{4 \beta-\alpha^{2} \eta}}{2}+C_{2} \sin \frac{\sqrt{4 \beta-\alpha^{2} \eta}}{2}}\right)}\right)\right]^{\frac{1}{s}}$, 
where $\eta=\mp \frac{s \omega}{\left(\tau \omega^{2}-1\right) \sqrt{\alpha^{2}-4 \beta}}(x-\omega t)$.

Substituting the general solutions of Eq. (5) into Eq. (13), we have the following two kinds of travelling wave solutions:

When $\alpha^{2}-4 \beta>0$,

$$
u(x, t)=\left[\frac{(s+1) \omega}{2 p} \pm \frac{(s+1) \omega}{2 p}\left(\frac{C_{1} \sinh \frac{\sqrt{\alpha^{2}-4 \beta} \eta}{2}+C_{2} \cosh \frac{\sqrt{\alpha^{2}-4 \beta} \eta}{2}}{C_{1} \cosh \frac{\sqrt{\alpha^{2}-4 \beta} \eta}{2}+C_{2} \sinh \frac{\sqrt{\alpha^{2}-4 \beta} \eta}{2}}\right)\right]^{\frac{1}{s}}
$$

where $\eta= \pm \frac{s \omega}{\left(\tau \omega^{2}-1\right) \sqrt{\alpha^{2}-4 \beta}}(x-\omega t)$.

When $\alpha^{2}-4 \beta<0$,

$$
u(x, t)=\left[\frac{(s+1) \omega}{2 p} \mp \frac{(s+1) \omega}{2 p} i\left(\frac{-C_{1} \sin \frac{\sqrt{4 \beta-\alpha^{2}} \eta}{2}+C_{2} \cos \frac{\sqrt{4 \beta-\alpha^{2}} \eta}{2}}{C_{1} \cos \frac{\sqrt{4 \beta-\alpha^{2}} \eta}{2}+C_{2} \sin \frac{\sqrt{4 \beta-\alpha^{2}} \eta}{2}}\right)\right]^{\frac{1}{s}}
$$

where $\eta= \pm \frac{s \omega}{\left(\tau \omega^{2}-1\right) \sqrt{\alpha^{2}-4 \beta}}(x-\omega t)$.

In Figs. 1, 2, 3 and 4, we show the effect of the time-delayed solution (14). It should be noted that when $\tau \rightarrow 0$, we can recover some traveling wave solutions of the generalized Burgers equation.

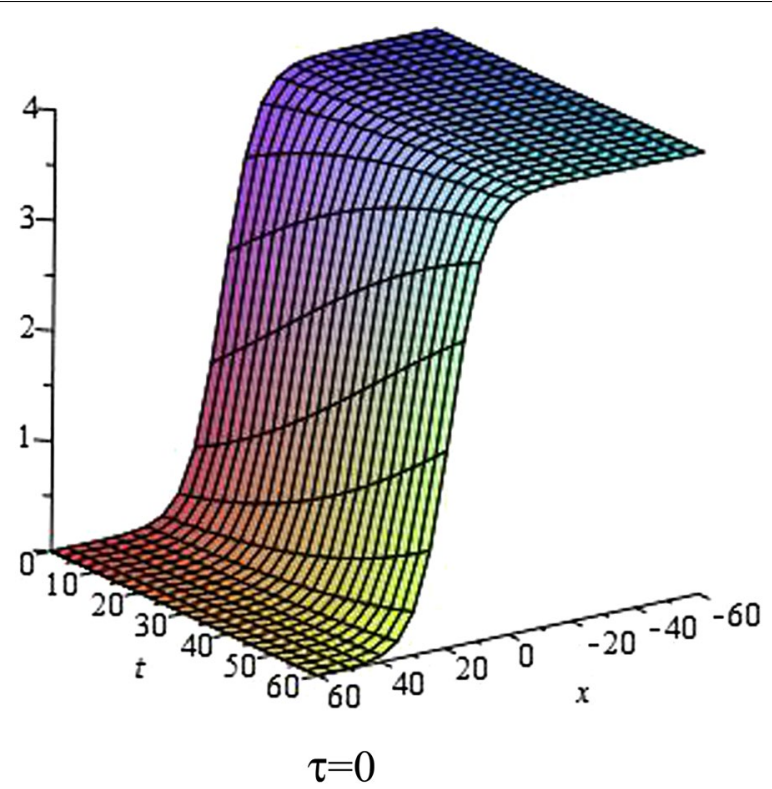

Fig. 1 The solution (14) for $\tau=0$ at $s=1, p=0.1, \omega=0.2, \alpha=5, \beta=4, t=1, C_{2}=0$ 


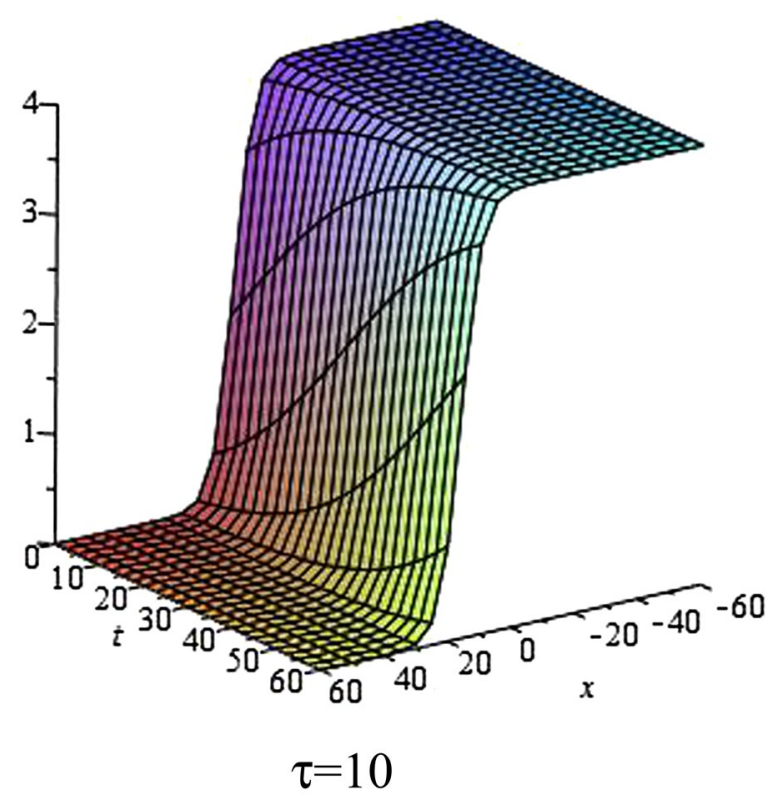

Fig. 2 The solution (14) for $\tau=10$ at $s=1, p=0.1, \omega=0.2, \alpha=5, \beta=4, t=1, C_{2}=0$

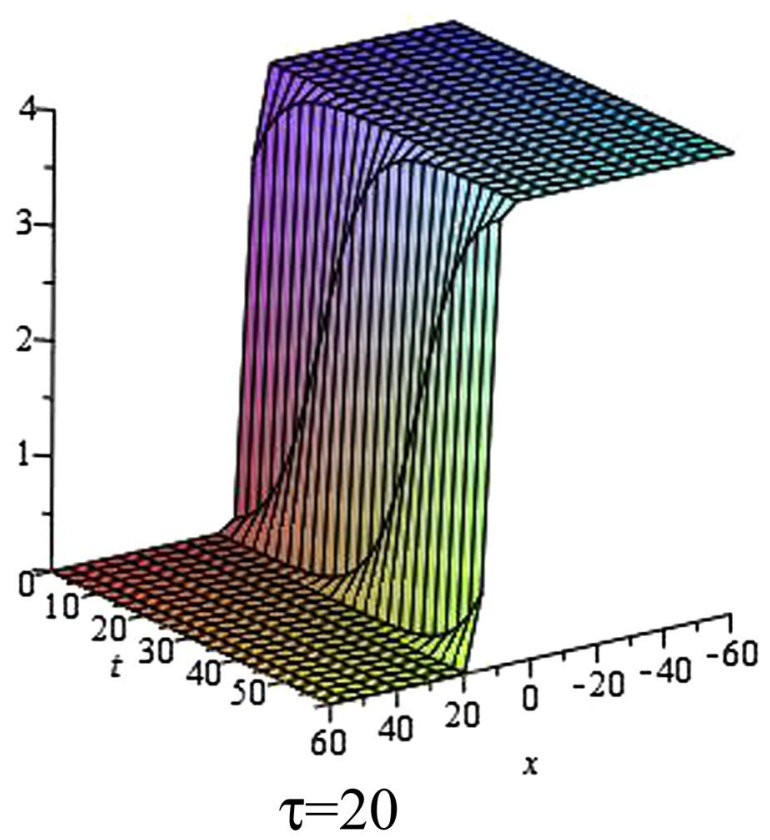

Fig. 3 The solution (14) for $\tau=20$ at $s=1, p=0.1, \omega=0.2, \alpha=5, \beta=4, t=1, C_{2}=0$

Solutions to the time-delayed generalized Burgers-Fisher equation

In this section, we consider the time-delayed generalized Burgers-Fisher equation:

$$
\tau v_{t t}+\left(1-\tau f_{v}\right) v_{t}=v_{x x}-p v^{s} v_{x}+f(v), \quad f(v)=q v\left(1-v^{s}\right), \quad q \neq 0 .
$$




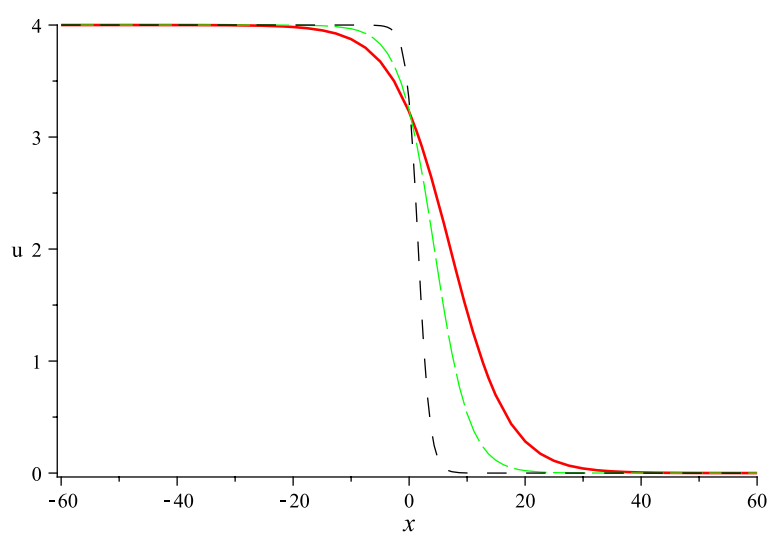

Fig. 4 The red, green and black lines represent the solution (14) for $\tau=0,10,20$ respectively at $s=1, p=0.1$, $\omega=0.2, \alpha=5, \beta=4, t=1, C_{2}=0$

By using the transformation

$$
v(x, t)=v(\eta), \quad \eta=k(x-\omega t)
$$

Equation (18) is converted into the following ordinary differential equation:

$$
k^{2}\left(\tau \omega^{2}-1\right) v^{\prime \prime}-k \omega\left(1-\tau q+(s+1) q v^{s}\right) v^{\prime}+p k v^{s} v^{\prime}-q v\left(1-v^{s}\right)=0 .
$$

Balancing $v^{\prime \prime}$ and $v^{s} v^{\prime}$ in Eq. (20) gives $n=\frac{1}{s}$. By using the transformation $v=W^{\frac{1}{s}}$, we convert Eq. (20) into

$$
\begin{aligned}
& k^{2}\left(\tau \omega^{2}-1\right)\left(\frac{1}{s}-1\right)\left(W^{\prime 2}+W W^{\prime \prime}\right)-k \omega(1-\tau q+(s+1) q W) W W^{\prime} \\
& \quad+p k v^{2} W^{\prime}-s q W^{2}(1-W)=0 .
\end{aligned}
$$

By balancing $W^{\prime 2}$ and $W^{2} W^{\prime}$ in Eq. (21), we suppose that Eq. (21) have the following solutions:

$$
W(\eta)=\sum_{l=-1}^{1} a_{l}\left(\frac{G^{\prime}}{G}\right)^{l} .
$$

Using the same procedure as in the previous example, we get a set of simultaneous algebraic equations for $a_{l}, k, \omega, \alpha$ and $\beta$. 


$$
\begin{aligned}
& \left(\frac{G^{\prime}}{G}\right)^{4}:-p k a_{1}^{3}+k \omega(s+1) q \tau a_{1}^{3}+\left(\tau \omega^{2}-1\right) k^{2} a_{1}^{2}\left(1+\frac{1}{s}\right)=0, \\
& \left(\frac{G^{\prime}}{G}\right)^{3}: k^{2}\left(\tau \omega^{2}-1\right)\left[2\left(1-\frac{1}{s}\right) \alpha a_{1}+a_{0}\right] a_{1}+k \omega(1-q \tau) a_{1}^{2} \\
& +(\omega(s+1)-p) k\left(2 a_{0}+\alpha a_{1}\right) a_{1}^{2}+s q a_{1}^{3}=0, \\
& \left(\frac{G^{\prime}}{G}\right)^{2}: \frac{k^{2}\left(\tau \omega^{2}-1\right) a_{1}}{s}\left(\left(\alpha^{2}+2 \beta\right) a_{1}+(2 s-1) a_{-1}+3 a_{0} \alpha s\right) \\
& -p k a_{1}\left(a_{-1} a_{1}+a_{0}+2 \alpha a_{0} a_{1}+a_{1}^{2} \beta\right)+k \omega(1-q \tau)\left(a_{0}+a_{1} \alpha\right) a_{1} \\
& +k \omega(s+1) q \tau a_{1}\left(a_{0}^{2}+a_{-1} a_{1}+2 a_{0} a_{1} \alpha+a_{1}^{2} \beta\right)+s q a_{1}^{2}\left(3 a_{0}-1\right)=0, \\
& \left(\frac{G^{\prime}}{G}\right)^{1}: \frac{k^{2}\left(\tau \omega^{2}-1\right) a_{1}}{s}\left[(8 s-1) a_{-1} \alpha+(2-s) a_{1} \beta \alpha+s\left(\alpha^{2}+\beta\right) a_{0}\right] \\
& +s q a_{1}\left(3 a_{-1} a_{1}+3 a_{0}^{2}-2 a_{0}\right)+k \omega(1-q \tau)\left(a_{0} a_{1} \alpha+a_{1}^{2} \beta\right) \\
& +p k a_{1}\left(-a_{-1} a_{1} \alpha-a_{0}^{2} \alpha+2 a_{-1} a_{0}-2 a_{0} a_{1} \beta\right)=0, \\
& \left(\frac{G^{\prime}}{G}\right)^{0}: k \omega(s+1) q \tau\left(a_{-1} a_{1}-a_{0}^{2}\right)\left(a_{1} \beta-a_{-1}\right)+s q\left(6 a_{-1} a_{0} a_{1}-2 a_{-1} a_{1}\right) \\
& -k \omega(1-q \tau) a_{0} a_{-1}+k \omega(1-q \tau) a_{1} \beta+p k\left(a_{-1}-a_{1} \beta\right)\left(a_{-1} a_{1}+a_{0}^{2}\right) \\
& +s q\left(-a_{0}^{2}+a_{0}^{3}\right)+\frac{k^{2}\left(\tau \omega^{2}-1\right)}{s}\left[4(2 s-1) a_{-1} a_{1} \beta+(5 s-2) a_{-1} a_{1} \alpha^{2}\right. \\
& \left.+(1-s)\left(a_{-1}^{2}+a_{1}^{2} \beta^{2}\right)+s \alpha a_{0}\left(a_{1} \beta+a_{-1}\right)\right]=0, \\
& \left(\frac{G^{\prime}}{G}\right)^{-1}:-k \omega(1-q \tau) a_{-1}\left(a_{-1}+a_{0} \alpha\right)+p k a_{-1}\left(2 a_{-1} a_{0}+a_{0}^{2} \alpha+a_{-1} a_{1} \alpha\right) \\
& +2 k^{2}\left(\tau \omega^{2}-1\right) \alpha a_{-1}\left(\frac{1}{s}-1\right)\left(a_{-1}-2 a_{1} \beta\right)+s q a_{-1}\left(3 a_{-1} a_{1}-2 a_{0}+3 a_{0}^{2}\right) \\
& +k^{2}\left(\tau \omega^{2}-1\right) a_{-1}\left(4 a_{1} \alpha \beta+2 a_{0} \beta+a_{0} \alpha^{2}+a_{-1} \alpha\right) \\
& -k \omega(s+1) q \tau a_{-1}\left(2 a_{-1} a_{0}+a_{-1} a_{1} \alpha+a_{0}^{2} \alpha\right)=0, \\
& \left(\frac{G^{\prime}}{G}\right)^{-2}: p k a_{-1}\left(a_{-1}^{2}+2 a_{-1} a_{0} \alpha+a_{-1} a_{1} \beta+a_{0}^{2} \beta\right)+q s a_{-1}^{2}\left(3 a_{0}-1\right) \\
& -k \omega(1-q \tau) a_{-1}\left(a_{-1} \alpha+a_{0} \beta+a_{-1}^{2}-a_{-1} a_{1} \beta\right) \\
& +\frac{k^{2}\left(\tau \omega^{2}-1\right) a_{-1}}{s}\left(2 a_{-1} \beta+2(2 s-1) a_{1} \beta^{2}+a_{-1} \alpha^{2}+3 a_{0} \alpha \beta s\right) \\
& -k \omega(s+1) q \tau a_{-1}\left(2 a_{-1} a_{0} \alpha+2 a_{-1} a_{1} \beta+a_{0}^{2} \beta\right)=0, \\
& \left(\frac{G^{\prime}}{G}\right)^{-3}: \frac{k^{2}\left(\tau \omega^{2}-1\right) a_{-1} \beta}{s}\left((s+2) a_{-1} \alpha+2 s a_{0} \beta\right)+q s a_{-1}^{3} \\
& +k a_{-1}^{2}\left(a_{-1} \alpha+2 a_{0} \beta\right)(p-\omega(s+1) q \tau)-k \omega(1-q \tau) a_{-1}^{2} \beta=0, \\
& \left(\frac{G^{\prime}}{G}\right)^{-4}: k^{2}\left(\tau \omega^{2}-1\right)\left(\frac{1}{s}+1\right) a_{-1}^{2} \beta^{2}+p k a_{-1}^{3} \beta-k \omega(s+1) q \tau a_{-1}^{3} \beta=0 \text {. }
\end{aligned}
$$

Solving the under-determined algebraic equations, we have the following results:

\section{Case 1}

$$
\begin{aligned}
& a_{-1}= \pm \frac{\beta}{\sqrt{\alpha^{2}-4 \beta}}, \quad a_{0}=\frac{1}{2} \pm \frac{\alpha}{2 \sqrt{\alpha^{2}-4 \beta}}, \quad a_{1}=0, \\
& k=\mp \frac{s(s+1)(1+q \tau) p}{\left(\tau p^{2}-(s+1)^{2}\right) \sqrt{\alpha^{2}-4 \beta}}, \quad \omega=\frac{p^{2}+(s+1)^{2} q}{p(s+1)(1+q \tau)} .
\end{aligned}
$$


Case 2

$$
\begin{array}{ll}
a_{-1}=0, \quad a_{0}=\frac{1}{2} \pm \frac{\alpha}{2 \sqrt{\alpha^{2}-4 \beta}}, & a_{1}= \pm \frac{1}{\sqrt{\alpha^{2}-4 \beta}} \\
k= \pm \frac{s(s+1)(1+q \tau) p}{\left(\tau p^{2}-(s+1)^{2}\right) \sqrt{\alpha^{2}-4 \beta}}, & \omega=\frac{p^{2}+(s+1)^{2} q}{p(s+1)(1+q \tau)} .
\end{array}
$$

By using Eqs. (23) and (24), expression (22) can be written as:

$$
\nu_{1}(\eta)=\frac{1}{2} \pm \frac{\alpha}{2 \sqrt{\alpha^{2}-4 \beta}} \pm \frac{\beta}{\sqrt{\alpha^{2}-4 \beta}}\left(\frac{G^{\prime}}{G}\right)^{-1}
$$

where $\eta=\mp \frac{s(s+1)(1+q \tau) p}{\left(\tau p^{2}-(s+1)^{2}\right) \sqrt{\alpha^{2}-4 \beta}}\left(x-\frac{p^{2}+(s+1)^{2} q}{p(s+1)(1+q \tau)} t\right)$.

$$
v_{2}(\eta)=\frac{1}{2} \pm \frac{\alpha}{2 \sqrt{\alpha^{2}-4 \beta}} \pm \frac{1}{\sqrt{\alpha^{2}-4 \beta}}\left(\frac{G^{\prime}}{G}\right),
$$

where $\eta= \pm \frac{s(s+1)(1+q \tau) p}{\left(\tau p^{2}-(s+1)^{2}\right) \sqrt{\alpha^{2}-4 \beta}}\left(x-\frac{p^{2} t+(s+1)^{2} q t}{p(s+1)(1+q \tau)}\right)$.

Substituting general solutions of Eq. (5) into Eqs. (25) and (26), we have two types of travelling wave solutions of the generalized time-delayed Burgers-Fisher equation as follows:

When $\alpha^{2}-4 \beta>0$,

$$
u(x, t)=\left[\frac{1}{\sqrt{\alpha^{2}-4 \beta}}\left(\frac{\sqrt{\alpha^{2}-4 \beta} \pm \alpha}{2} \pm \frac{2 \beta}{-\alpha+\sqrt{\alpha^{2}-4 \beta}\left(\frac{C_{1} \sinh \frac{\sqrt{\alpha^{2}-4 \beta} \eta}{2}+C_{2} \cosh \frac{\sqrt{\alpha^{2}-4 \beta} \eta}{2}}{C_{1} \cosh \frac{\sqrt{\alpha^{2}-4 \beta} \eta}{2}+C_{2} \sinh \frac{\sqrt{\alpha^{2}-4 \beta} \eta}{2}}\right)}\right)\right]^{\frac{1}{s}},
$$

where $\eta=\mp \frac{s(s+1)(1+q \tau) p}{\left(\tau p^{2}-(s+1)^{2}\right) \sqrt{\alpha^{2}-4 \beta}}\left(x-\frac{p^{2} t+(s+1)^{2} q t}{p(s+1)(1+q \tau)}\right)$

$$
u(x, t)=\left[\frac{1}{2} \pm \frac{1}{2}\left(\frac{C_{1} \sinh \frac{\sqrt{\alpha^{2}-4 \beta} \eta}{2}+C_{2} \cosh \frac{\sqrt{\alpha^{2}-4 \beta} \eta}{2}}{C_{1} \cosh \frac{\sqrt{\alpha^{2}-4 \beta} \eta}{2}+C_{2} \sinh \frac{\sqrt{\alpha^{2}-4 \beta} \eta}{2}}\right)\right]^{\frac{1}{s}}
$$

where $\eta= \pm \frac{s(s+1)(1+q \tau) p}{\left(\tau p^{2}-(s+1)^{2}\right) \sqrt{\alpha^{2}-4 \beta}}\left(x-\frac{p^{2}+(s+1)^{2} q}{p(s+1)(1+q \tau)} t\right)$.

When $\alpha^{2}-4 \beta<0$,

$$
u(x, t)=\left[\frac{1}{\sqrt{\alpha^{2}-4 \beta}}\left(\frac{\sqrt{\alpha^{2}-4 \beta} \pm \alpha}{2} \pm \frac{2 \beta}{-\alpha+\sqrt{\alpha^{2}-4 \beta}\left(\frac{-C_{1} \sin \frac{\sqrt{4 \beta-\alpha^{2}} \eta}{2}+C_{2} \cos \frac{\sqrt{4 \beta-\alpha^{2}} \eta}{2}}{C_{1} \cos \frac{\sqrt{4 \beta-\alpha^{2}} \eta}{2}+C_{2} \sin \frac{\sqrt{4 \beta-\alpha^{2} \eta}}{2}}\right)}\right)\right]^{\frac{1}{s}},
$$


where $\eta=\mp \frac{s(s+1)(1+q \tau) p}{\left(\tau p^{2}-(s+1)^{2}\right) \sqrt{\alpha^{2}-4 \beta}}\left(x-\frac{p^{2}+(s+1)^{2} q}{p(s+1)(1+q \tau)} t\right)$.

$$
u(x, t)=\left[\frac{1}{2} \mp \frac{i}{2}\left(\frac{-C_{1} \sin \frac{\sqrt{4 \beta-\alpha^{2}} \eta}{2}+C_{2} \cos \frac{\sqrt{4 \beta-\alpha^{2}} \eta}{2}}{C_{1} \cos \frac{\sqrt{4 \beta-\alpha^{2}} \eta}{2}+C_{2} \sin \frac{\sqrt{4 \beta-\alpha^{2}} \eta}{2}}\right)\right]^{\frac{1}{s}},
$$

where $\eta= \pm \frac{s(s+1)(1+q \tau) p}{\left(\tau p^{2}-(s+1)^{2}\right) \sqrt{\alpha^{2}-4 \beta}}\left(x-\frac{p^{2}+(s+1)^{2} q}{p(s+1)(1+q \tau)} t\right)$.

In Figs. 5, 6, 7 and 8, we show the effect of the time-delayed solution (27). It should be noted that when $\tau \rightarrow 0$, we can recover some traveling wave solutions of the generalized Burgers-Fisher equation.

Remark 1 By using extended $\left(\frac{G^{\prime}}{G}\right)$-expansion method, we can obtain solutions including all the solutions given in Deng et al. (2009) as special cases. For example, if setting $C_{2}=0$, then solution (28) is the same as Eq. (19) in Deng et al. (2009). Similarly, solution (28) is also the same as Eq. (20) obtained in Deng et al. (2009) when we set $C_{1}=0$. It shows that extended $\left(\frac{G^{\prime}}{G}\right)$-expansion method is more powerful than the method in Deng et al. (2009) in constructing exact solutions.

Remark 2 Rosa et al. (2015) applied Lie classical method and $\left(\frac{G^{\prime}}{G}\right)$-expansion method to Fisher equation and derived some new traveling wave solutions. If setting $a_{l}(l=-n \ldots-1)=0$, then Eq. (4) becomes Eq. (14) in Rosa and Gandarias, (2015). So if we applied Lie classical method and extended $\left(\frac{G^{\prime}}{G}\right)$-expansion method to Fisher equation, then many more exact solutions can be obtained. Searching exact solutions by use of Lie classical method and extended $\left(\frac{G^{\prime}}{G}\right)$-expansion method is our future work.

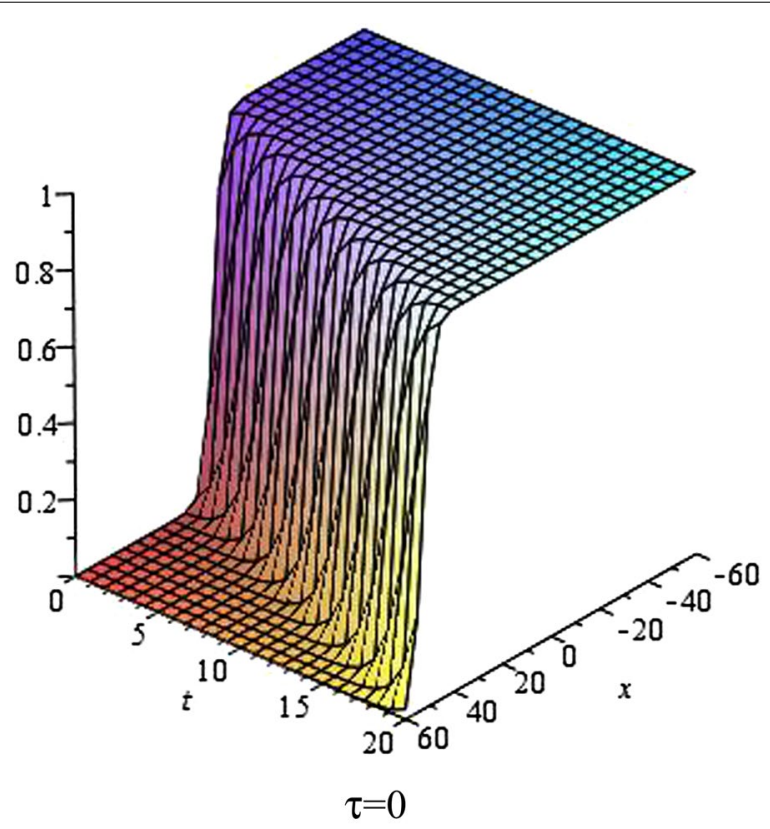

Fig. 5 The solution (27) for $\tau=0$ at $p=q=s=1, \alpha=5, \beta=4, t=1, C_{2}=0$ 


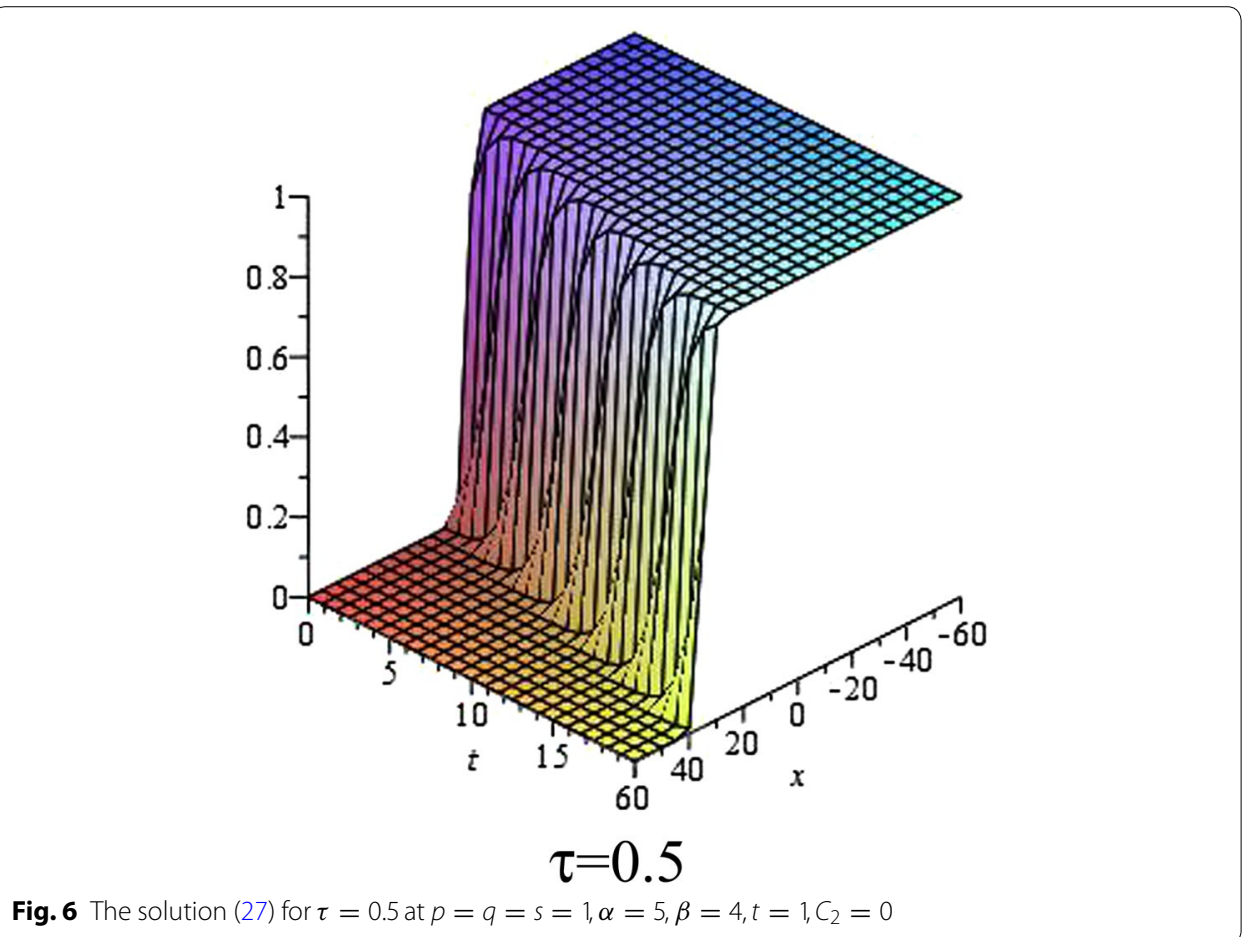

Fig. 6 The solution (27) for $\tau=0.5$ at $p=q=s=1, \alpha=5, \beta=4, t=1, C_{2}=0$

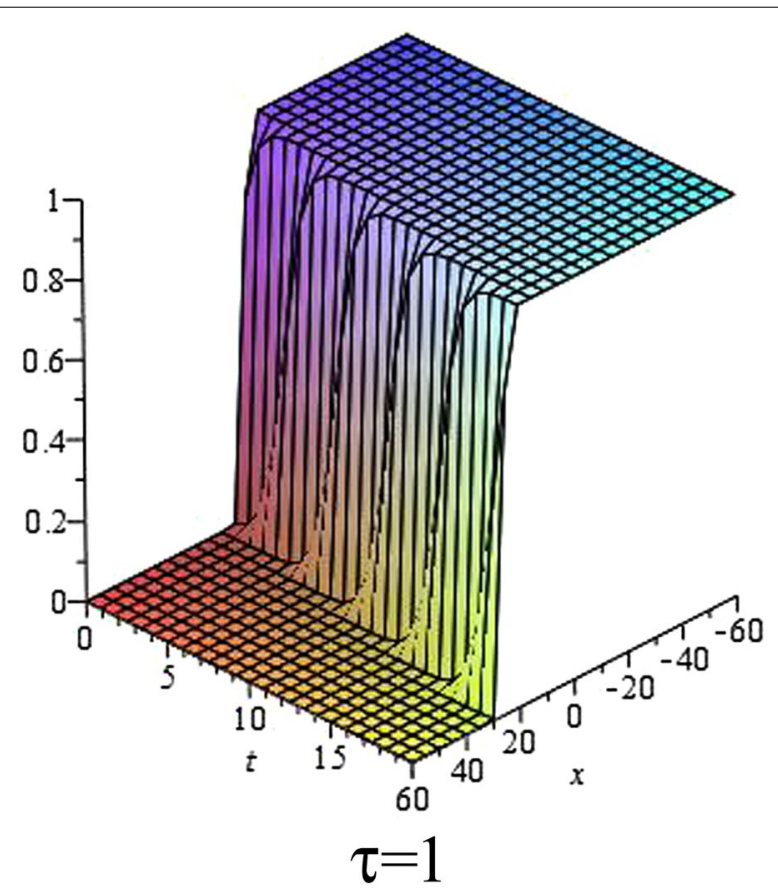

Fig. 7 The solution (27) for $\tau=1$ at $p=q=s=1, \alpha=5, \beta=4, t=1, C_{2}=0$ 


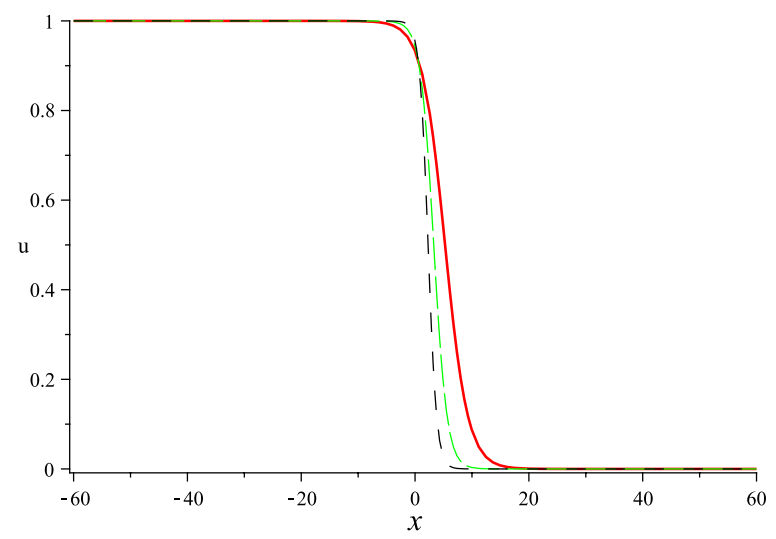

Fig. 8 The red, green and black lines represent the solution (27) for $\tau=0,0.5,1$ respectively at $p=q=s=1$, $\alpha=5, \beta=4, t=1, C_{2}=0$

\section{Conclusions}

Based on the extended $\left(\frac{G^{\prime}}{G}\right)$-expansion method, we have constructed many traveling wave solutions of the time-delayed generalized Burgers-type equation which include the hyperbolic function solutions, trigonometric function solutions. The results show that the proposed method is very effective and can be used to handling many other nonlinear time-delayed evolution equations.

\section{Declarations}

In this section, we illustrate how to get the solutions presented after Eq. (5).

The general solutions of Eq. (5) can easily obtained as follows:

$$
G(\eta)= \begin{cases}a_{1} e^{\frac{-\alpha+\sqrt{\alpha^{2}-4 \beta}}{2} \eta}+a_{2} e^{\frac{-\alpha-\sqrt{\alpha^{2}-4 \beta}}{2} \eta}, & \alpha^{2}-4 \beta>0, \\ e^{-\frac{\alpha}{2} \eta}\left(a_{1} \cos \frac{\sqrt{4 \beta-\alpha^{2}} \eta}{2}+a_{2} \sin \frac{\sqrt{4 \beta-\alpha^{2}} \eta}{2}\right), & \alpha^{2}-4 \beta<0 . \\ \left(a_{1}+a_{2} \eta\right) e^{-\frac{\alpha}{2} \eta}, & \alpha^{2}-4 \beta=0 .\end{cases}
$$

When $\alpha^{2}-4 \beta>0$

$$
G^{\prime}(\eta)=\frac{-\alpha+\sqrt{\alpha^{2}-4 \beta}}{2} a_{1} e^{\frac{-\alpha+\sqrt{\alpha^{2}-4 \beta}}{2} \eta}+\frac{-\alpha-\sqrt{\alpha^{2}-4 \beta}}{2} a_{2} e^{\frac{-\alpha-\sqrt{\alpha^{2}-4 \beta}}{2} \eta},
$$

then

$$
\begin{aligned}
& \frac{G^{\prime}(\eta)}{G(\eta)}=\frac{\frac{-\alpha+\sqrt{\alpha^{2}-4 \beta}}{2} a_{1} e^{\frac{\sqrt{\alpha^{2}-4 \beta}}{2} \eta}+\frac{-\alpha-\sqrt{\alpha^{2}-4 \beta}}{2} a_{2} e^{\frac{-\sqrt{\alpha^{2}-4 \beta}}{2} \eta}}{a_{1} e^{\frac{-\alpha+\sqrt{\alpha^{2}-4 \beta}}{2} \eta}+a_{2} e^{\frac{-\alpha-\sqrt{\alpha^{2}-4 \beta}}{2} \eta}} \\
& =-\frac{\alpha}{2}+\frac{\sqrt{\alpha^{2}-4 \beta}}{2} \frac{a_{1} e^{\frac{\sqrt{\alpha^{2}-4 \beta}}{2} \eta}-a_{2} e^{\frac{-\sqrt{\alpha^{2}-4 \beta}}{2} \eta}}{a_{1} e^{\frac{\sqrt{\alpha^{2}-4 \beta}}{2} \eta}+a_{2} e^{\frac{-\sqrt{\alpha^{2}-4 \beta}}{2} \eta}}
\end{aligned}
$$


Taking $C_{1}=\frac{a_{1}+a_{2}}{2}, C_{2}=\frac{a_{1}-a_{2}}{2}, r=\frac{\sqrt{\alpha^{2}-4 \beta}}{2} \eta$, we can convert Eq. (31) into the following form:

$$
\begin{aligned}
\frac{G^{\prime}(\eta)}{G(\eta)} & =-\frac{\alpha}{2}+\frac{\sqrt{\alpha^{2}-4 \beta}}{2} \frac{\left(C_{1}+C_{2}\right) e^{\frac{\sqrt{\alpha^{2}-4 \beta}}{2} \eta}-\left(C_{1}-C_{2}\right) e^{\frac{-\sqrt{\alpha^{2}-4 \beta}}{2} \eta}}{\left(C_{1}+C_{2}\right) e^{\frac{\sqrt{\alpha^{2}-4 \beta}}{2} \eta}+\left(C_{1}-C_{2}\right) e^{\frac{-\sqrt{\alpha^{2}-4 \beta}}{2} \eta}} \\
& =-\frac{\alpha}{2}+\frac{\sqrt{\alpha^{2}-4 \beta}}{2} \frac{C_{1}\left(e^{r}-e^{-r}\right)-C_{2}\left(e^{r}+e^{-r}\right)}{C_{1}\left(e^{r}+e^{-r}\right)+C_{2}\left(e^{r}-e^{-r}\right)} \\
& =-\frac{\alpha}{2}+\frac{\sqrt{\alpha^{2}-4 \beta}}{2} \frac{C_{1}\left(\frac{e^{r}-e^{-r}}{2}\right)-C_{2}\left(\frac{e^{r}+e^{-r}}{2}\right)}{C_{1}\left(\frac{e^{r}+e^{-r}}{2}\right)+C_{2}\left(\frac{e^{r}-e^{-r}}{2}\right)} \\
& =-\frac{\alpha}{2}+\frac{\sqrt{\alpha^{2}-4 \beta}}{2}\left(\frac{C_{1} \sinh \left(\frac{\sqrt{\alpha^{2}-4 \beta} \eta}{2}\right)+C_{2} \cosh \left(\frac{\sqrt{\alpha^{2}-4 \beta} \eta}{2}\right)}{C_{1} \cosh \left(\frac{\sqrt{\alpha^{2}-4 \beta} \eta}{2}\right)+C_{2} \sinh \left(\frac{\sqrt{\alpha^{2}-4 \beta} \eta}{2}\right)}\right) .
\end{aligned}
$$

When $\alpha^{2}-4 \beta<0$

$$
\begin{aligned}
G^{\prime}(\eta)= & -\frac{\alpha}{2} e^{\frac{-\alpha}{2} \eta}\left(a_{1} \cos \frac{\sqrt{4 \beta-\alpha^{2}} \eta}{2}+a_{2} \sin \frac{\sqrt{4 \beta-\alpha^{2}} \eta}{2}\right) \\
& +\frac{\sqrt{4 \beta-\alpha^{2}} \eta}{2} e^{\frac{-\alpha}{2} \eta}\left(-a_{1} \sin \frac{\sqrt{4 \beta-\alpha^{2}} \eta}{2}+a_{2} \cos \frac{\sqrt{4 \beta-\alpha^{2}} \eta}{2}\right)
\end{aligned}
$$

then

$$
\begin{aligned}
\frac{G^{\prime}(\eta)}{G(\eta)}= & \frac{-\frac{\alpha}{2} e^{\frac{-\alpha}{2} \eta}\left(a_{1} \cos \frac{\sqrt{4 \beta-\alpha^{2}} \eta}{2}+a_{2} \sin \frac{\sqrt{4 \beta-\alpha^{2}} \eta}{2}\right)}{e^{-\frac{\alpha}{2} \eta}\left(a_{1} \cos \frac{\sqrt{4 \beta-\alpha^{2}} \eta}{2}+a_{2} \sin \frac{\sqrt{4 \beta-\alpha^{2}} \eta}{2}\right)} \\
& +\frac{\frac{\sqrt{4 \beta-\alpha^{2}} \eta}{2} e^{\frac{-\alpha}{2} \eta}\left(-a_{1} \sin \frac{\sqrt{4 \beta-\alpha^{2}} \eta}{2}+a_{2} \cos \frac{\sqrt{4 \beta-\alpha^{2}} \eta}{2}\right)}{e^{-\frac{\alpha}{2} \eta}\left(a_{1} \cos \frac{\sqrt{4 \beta-\alpha^{2}} \eta}{2}+a_{2} \sin \frac{\sqrt{4 \beta-\alpha^{2}} \eta}{2}\right)}
\end{aligned}
$$

Taking $C_{1}=a_{1}, C_{2}=a_{2}$, we can convert Eq. (32) into the following form:

$$
\frac{G^{\prime}(\eta)}{G(\eta)}=-\frac{\alpha}{2}+\frac{\sqrt{4 \beta-\alpha^{2}}}{2}\left(\frac{-C_{1} \sin \left(\frac{\sqrt{4 \beta-\alpha^{2}} \eta}{2}\right)+C_{2} \cos \left(\frac{\sqrt{4 \beta-\alpha^{2}} \eta}{2}\right)}{C_{1} \cos \left(\frac{\sqrt{4 \beta-\alpha^{2}} \eta}{2}\right)+C_{2} \sin \left(\frac{\sqrt{4 \beta-\alpha^{2}} \eta}{2}\right)}\right)
$$

When $\alpha^{2}-4 \beta=0$

$$
G^{\prime}(\eta)=\left[a_{2}-\left(a_{1}+a_{2} \eta\right) \frac{\alpha}{2}\right] e^{-\frac{\alpha}{2} \eta},
$$


then

$$
\begin{aligned}
\frac{G^{\prime}(\eta)}{G(\eta)} & =\frac{\left[a_{2}-\left(a_{1}+a_{2} \eta\right) \frac{\alpha}{2}\right] e^{-\frac{\alpha}{2} \eta}}{\left(a_{1}+a_{2} \eta\right) e^{-\frac{\alpha}{2} \eta}} \\
& =-\frac{\alpha}{2}+\frac{a_{2}}{a_{1}+a_{2} \eta}
\end{aligned}
$$

Taking $C_{1}=a_{1}, C_{2}=a_{2}$, we can convert Eq. (33) into the following form:

$$
\frac{G^{\prime}(\eta)}{G(\eta)}=-\frac{\alpha}{2}+\frac{C_{2}}{C_{1}+C_{2} \eta}
$$

Authors' contributions

BT, YZF, XMW, JXW and SJC, with the consultation of each other performed this research and drafted the manuscript together. All authors read and approved the final manuscript.

\section{Author details}

${ }^{1}$ School of Mathematics and Computer Science, Hubei University of Arts and Science, Xiangyang 441053, China.

${ }^{2}$ School of Mathematics and Statistics, Xi'an Jiaotong University, Xi'an 710049, China. ${ }^{3}$ School of Mathematics and Statistics, Wuhan University, Wuhan 430072, China. ${ }^{4}$ Department of Mechanical Engineering, University of Texas at Dallas, Richardson, TX 75080, USA.

\section{Acknowledgements}

The authors would like to extend sincere gratitude to the referee for carefully reading and useful suggestions to improve the paper.

Competing interests

The authors declare that they have no competing interests.

\section{Funding}

This research work is supported by the National Natural Science Foundation of China $(11526088,11501186)$ and Natural Science Foundation of Hubei Province (2014CFB640).

Received: 15 April 2016 Accepted: 30 November 2016

Published online: 12 December 2016

\section{References}

Abdel Rady AS, Osman ES, Khalfallah M (2010) The homogeneous balance method and its application to the BenjaminBona-Mahoney (BBM) equation. Appl Math Comput 217:1385-1390

Deng XJ, Han LB, Li X (2009) Travelling solitary wave solutions for generalized time-delayed Burgers-Fisher equation. Commun Theor Phys 52:284-286

Eslami M, Fathi vajargah B, Mirzazadeh M (2014) Exact solutions of modified Zakharov-Kuznetsov equation by the homogeneous balance method. Ain Shams Eng J 5:221-225

Fan E (2000) Two new applications of the homogeneous balance method. Phys Lett A 265:353-357

Fan E, Zhang HQ (1998a) New exact solutions to a system of coupled KdV equations. Phys Lett A 245:389-392

Fan EG, Zhang HQ (1998b) A note on the homogeneous balance method. Phys Lett A 246:403-406

He JH (1997) A new approach to nonlinear partial differential equations. Commun Nonlinear Sci Numer Simul 2:230-235 He JH (1999) Homotopy perturbation technique. Comput Methods Appl Mech Eng 178:257-262

He JH, Wu XH (2007) Variational iteration method: new development and applications. Comput Math Appl 54:881-894 Islam MH, Khan K, Akbar MA, Salam MA (2014) Exact traveling wave solutions of modified KdV-Zakharov-Kuznetsov equation and viscous Burgers equation. SpringerPlus 3:105

Kar S, Banik SK, Ray DS (2003) Exact solutions of Fisher and Burgers equations with finite transport memory. J Phys A Math Gen 36:2771-2780

Khan K, Akbar MA (2014) Study of analytical method to seek for exact solutions of variant Boussinesq equations. SpringerPlus 3:324

Kumar S (2014) A new analytical modelling for telegraph equation via Laplace transform. Appl Math Model 38:3154-3163 Kumar S, Singh OP (2009) Numerical inversion of the Abel integral equation using homotopy perturbation method. Z Naturforschung 65a:677-682

Kumar S, Khan Y, Yildirim A (2012) A mathematical modelling arising in the chemical system and its approximate numerical solution. Asia Pac J Chem Eng 7(6):835-840

Kumar S, Kumar D, Singh J (2016a) Fractional modelling arising in unidirectional propagation of long waves in dispersive media. Nonlinear Anal Adv. doi:10.1515/anona-2013-0033

Kumar S, Kumar A, Baleanu D (2016b) Two analytical method for time-fractional nonlinear coupled Boussinesq-Burger equations arises in propagation of shallow water waves. Nonlinear Dyn. doi:10.1007/s11071-016-2716-2

Maple. www.maplesoft.com 
Rosa M, Bruzón M, Gandarias MDLL (2015) Lie symmetries and conservation laws of a Fisher equation with nonlinear convection term. Discrete Cont Dyn Syst S 8(6):1331-1339

Senthilvelan M (2001) On the extended applications of homogenous balance method. Appl Math Comput 123:381-388

Sirendaoreji SJ (2003) Auxiliary equation method for solving nonlinear partial differential equations. Phys Lett A 309:387-396

Tang B, He Y, Wei L, Wang S (2011) Variable-coefficient discrete (G'/G)-expansion method for nonlinear differential-difference equations. Phys Lett A 375:3355-3361

Tang B, Wang X, Wei L, Zhang X (2014) Exact solutions of fractional heat-like and wave-like equations with variable coefficients. Int J Numer Methods Heat Fluid Flow 24:455-467

Tang B, Wang X, Fan Y, Qu J (2016) Exact solutions for a generalized KdV-MKdV equation with variable coefficients. Math Probl Eng 2016:5274243. doi:10.1155/2016/5274243

Wang ML, Li XZ, Zhang JL (2008) The (G'/G)-expansion method and traveling wave solutions of nonlinear evolution equations in mathematical physics. Phys Lett A 372:417-423

Yao J, Kumar A, Kumar S (2015) A fractional model to describing the Brownian motion of particles and its analytical solution. Adv Mech Eng 7(12):1-11

Yin XB, Kumar S, Kumar D (2015) A modified homotopy analysis method for solution of fractional wave equations. Adv Mech Eng 7(12):1-8

Yomba E (2004) Construction of new soliton-like solutions for the $(2+1)$ dimensional KdV equation with variable coefficients. Chaos Solitons Fractals 21:75-79

Zhang J, Jiang F, Zhao X (2010) An improved (G'/G)-expansion method for solving nonlinear evolution equations. Int J Comput Math 87:1716-1725

Zhao XQ, Tang DG (2002) A new note on a homogeneous balance method. Phys Lett A 297:59-67

\section{Submit your manuscript to a SpringerOpen ${ }^{\circ}$ journal and benefit from:}

- Convenient online submission

- Rigorous peer review

- Immediate publication on acceptance

- Open access: articles freely available online

- High visibility within the field

- Retaining the copyright to your article

Submit your next manuscript at $\boldsymbol{\nabla}$ springeropen.com 